PoS $\quad \begin{aligned} & \text { PROCEEDINGS } \\ & \text { OF SCIENCE }\end{aligned}$

\title{
Review of charged Higgs searches at the Tevatron
}

\section{Phillip GUTIERREZ ${ }^{* \dagger}$}

University of Oklahoma

E-mail: pgutierrez@ou.edu

We discuss searches for charged Higgs bosons performed by the CDF and D0 collaborations at the Fermilab Tevatron in the mass range of 80 to $300 \mathrm{GeV}$.

Prospects for Charged Higgs Discovery at Colliders

27-30 September 2010

Uppsala University, Sweden

\footnotetext{
* Speaker.

${ }^{\dagger}$ For the CDF and D0 Collaborations
} 


\section{Introduction}

Although the standard model of particle physics (SM) is remarkably successful at describing the fundamental particles and their interactions, the mechanism for the breaking of elecroweak symmetry (EWSB) has yet to be confirmed. In the SM, the EWSB sector consists of four scalar fields represented by a single $S U(2)$ complex doublet [迆]. Following EWSB, three of the fields are responsible for the generation of the $W^{ \pm}$and $Z$ masses, while the fourth is the neutral Higgs boson. At the present time, the Tevatron experiments have set 95\% CL exclusion limits on the mass of the SM Higgs boson for the ranges 100 to $109 \mathrm{GeV}$ and 158 to $175 \mathrm{GeV}$ [2]. In addition to these constraints on the SM Higgs boson, the Tevatron experiments have also set limits on neutral and charged Higgs bosons $\left(H^{ \pm}\right)$in the context of several models beyond the SM.

In this review, we discuss recent results from searches for $H^{ \pm}$performed by the two Fermilab Tevatron collaborations, CDF [3] and D0 [印, with data from $p \bar{p}$ collisions at a center of mass energy $\sqrt{s}=1.96 \mathrm{TeV}$. The searches cover a mass range from 60 to $300 \mathrm{GeV}$, using data samples corresponding to 1 to $2.7 \mathrm{fb}^{-1}$ of integrated luminosity. These searches are categorized as indirect and direct. Indirect searches seek deviations from expectations of the SM to infer production of $H^{ \pm}$. Direct searches refer to searches for resonant enhancements or evidence consistent with $H^{ \pm}$decays. Most of the searches are carried out in the context of a two-Higgs doublet model (THDM), where an additional Higgs doublet is added to the SM Higgs sector [5], thereby introducing four additional fields, two that are neutral and two that are singly charged. A priori, the fermion couplings to the two doublets are not specified. The only requirements are that no flavor-changing neutral currents (FCNC) appear at leading order in EW couplings and that the value of $\rho$ is $\approx 1$, where $\rho=M_{W}^{2} / M_{Z}^{2} \cos ^{2} \theta_{W}$, with $M_{W}$ and $M_{Z}$ being the $W$ and $Z$ masses, and $\theta_{W}$ is the weak mixing angle, respectively. The couplings can therefore be selected on the basis of a theorem by Glashow and Weinberg [6] that states: if all fermions of a given electric charge couple to the same doublet, FCNC are absent at leading order. This leads to two possibilities [5]: 1) Type-1 models where all the quarks and leptons couple to a single doublet, and 2) Type-2 models, where the up-type quarks and leptons couple to one doublet and the down-type quarks and leptons couple to the other doublet, which is the same requirement as in the minimal supersymmetric standard model (MSSM) that is required to avoid anomalies [5]. The parameters of interest in Type 1 and 2 models are the mass of the $H^{ \pm}\left(M_{H^{ \pm}}\right)$and $\tan \beta$ (the ratio of vacuum expectation values of the two Higgs doublets). An alternative to applying the Glashow-Weinberg theorem [6] are available in Type-3 models that select the parameters in a manner that suppresses FCNC at lowest order and admits coupling of all quarks and leptons to either one or both Higgs doublets. The Type-3 model described in Ref. [7], which suppresses FCNC because of the small quark masses of the two lightest flavor generations, is considered by D0 in the $H^{+} \rightarrow t \bar{b}$ search. The $H^{ \pm}$analyses described below all assume charge conjugation invariance, and are carried out under the assumptions that branching ratios $\operatorname{BR}(t \rightarrow$ $\left.W^{+} b\right)+\mathrm{BR}\left(t \rightarrow H^{+} b\right)=1$, and that $M_{H^{ \pm}}<m_{t}+m_{b}$, with the exception of the D0 search for $H^{+} \rightarrow t \bar{b}$.

\section{Indirect Searches}

The indirect searches look for inconsistencies in branching ratios for different observed $t \bar{t}$ 
final states. For example, if $\mathrm{BR}\left(t \rightarrow H^{+} b\right)$ is finite, and $\mathrm{BR}\left(H^{+} \rightarrow \tau^{+} v_{\tau}\right) \approx 1$, then the number of $t \bar{t} \rightarrow \ell^{ \pm}+$jets events, where $\ell^{ \pm}$represents either an electron $\left(e^{ \pm}\right)$or a muon $\left(\mu^{ \pm}\right)$from the decay of the $W$, will be less than expected in the SM. On the other hand, the number of $t \bar{t} \rightarrow \tau^{ \pm}+$jets events will be greater than SM expectations. Several techniques have been employed at the Tevatron to carry out such indirect searches, and we here review the most recent of these.

\subsection{CDF: MSSM Search}

The CDF collaboration has performed a search for $H^{ \pm}$in the context of the MSSM using $193 \mathrm{pb}^{-1}$ of integrated luminosity [8]. The analysis uses previous measurements of the $t \bar{t}$ production cross section $\left(\sigma_{t \bar{t}}\right)$ in the $\ell^{ \pm}+\not_{T}+$ jets $+X$ final states, where $X$ is either a $\ell^{ \pm}$, a $\tau$ lepton that decays into hadrons $+v_{\tau}\left(\tau_{h}\right)$, or one or more $b$-tagged jets, and $E_{T}$ is the momentum imbalance transverse to the beam axis. The individual data samples are required to be orthogonal to avoid correlations among all the final states.

The analysis considers the decays: $H^{+} \rightarrow \tau v_{\tau}, H^{+} \rightarrow c \bar{s}, H^{+} \rightarrow t^{*} \bar{b}$, and $H^{+} \rightarrow W^{+} h^{0} \rightarrow$ $W^{+} b \bar{b}$. The branching fractions for these decays include radiative corrections using the program CPSUPERH [9] for different values of $M_{H^{ \pm}}, \tan \beta$, and MSSM symmetry-breaking parameters [8]. To set the 95\% CL Bayesian exclusion limits, CDF assumes a prior probability density that is "flat" in $\log (\tan \beta)$, with efficiencies for different $H^{ \pm}$masses and decay modes based on a simulation of the CDF detector using events generated by the PYTHIA [10] Monte Carlo (MC) generator. Figure 11 (a) shows the region of exclusion for $M_{H^{ \pm}}$and $\tan \beta$.

To reduce the model dependence of the exclusion on $\mathrm{BR}\left(t \rightarrow H^{+} b\right)$, the five possible decays were divided into 21 bins in $\mathrm{BR}\left(H^{+} \rightarrow X\right)$ for each mass, subject to the constraints $\sum_{i} \mathrm{BR}\left(H^{+} \rightarrow\right.$ $\left.X_{i}\right)=1, \mathrm{BR}\left(h^{0} \rightarrow b \bar{b}\right)=0.9$ and $m_{h}=70 \mathrm{GeV}$. Limits were calculated for each BR combination at a given $M_{H^{ \pm}}$, and the least restrictive limit for each $M_{H^{ \pm}}$is shown in Fig. 11 (b).
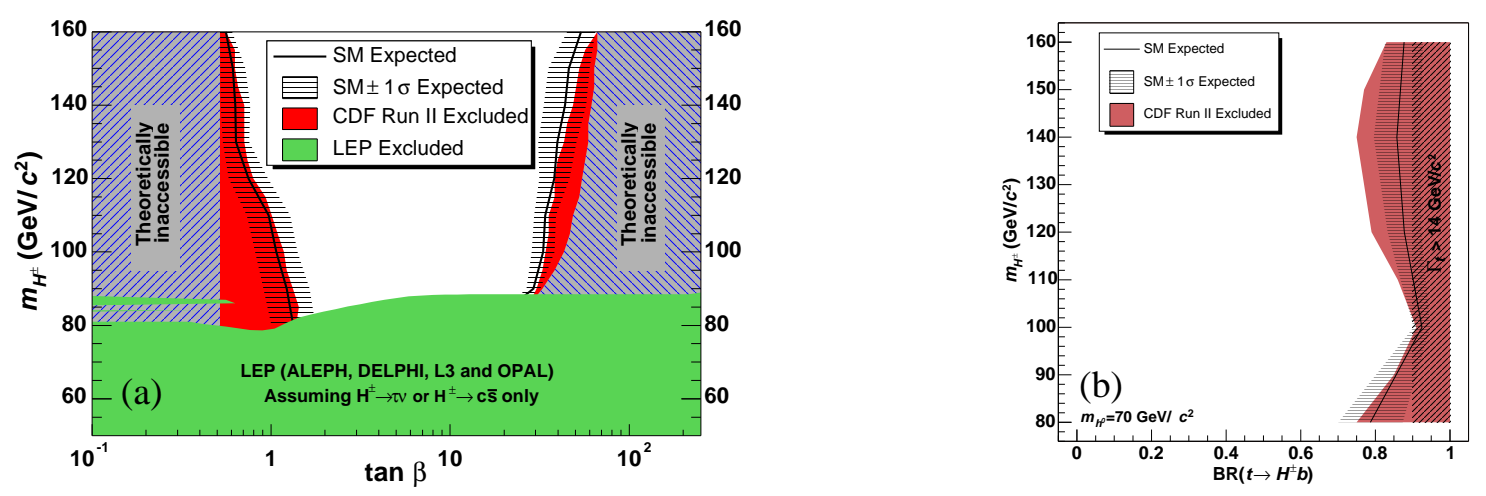

Figure 1: (a) The region of $H^{ \pm}$exclusion based on the MSSM parameters of Ref. [8]. Other MSSM scenarios are discussed in Ref. [1]. (b) The exclusion region for $\mathrm{BR}\left(t \rightarrow H^{+} b\right)$ using the model-independent approach described in the text.

\subsection{D0: Topological}

The D0 collaboration has extracted a charged Higgs limit for $\operatorname{BR}\left(H^{+} \rightarrow \tau^{+} v_{\tau}\right)=1$ (tauonic decays) [12], using the same data sample and event selections used in the "topological" measurement of $\sigma_{t \bar{t}}$ in the $\ell^{ \pm}+$jets final state using $0.9 \mathrm{fb}^{-1}$ of integrated luminosity [13]. The data are 
separated into two event categories of 3 and $>3$ jets to improve signal discrimination. To differentiate between $t \bar{t}$ and background events, a multivariate discriminant is defined by: $\mathcal{D}(\mathbf{x})=$ $p(\mathbf{x} \mid S) /\{p(\mathbf{x} \mid S)+p(\mathbf{x} \mid \mathcal{B})\}$, where $p$ is the probability density for a set of observables $\mathbf{x}$, given contributions from background $\mathcal{B}$ and signal $S$. The signal is $t \bar{t}$ events, which includes $t$ quarks that decay to $H^{ \pm}$and therefore depends on $\mathrm{BR}\left(t \rightarrow H^{+} b\right)$ and $M_{H^{ \pm}}$. The background includes all important sources, but is dominated by $W+$ jets and multijet events as can be seen in Fig. 22 (a) and (b). For a $\operatorname{BR}\left(t \rightarrow H^{ \pm} b\right)=0$ in Fig. 2 (a), the data agree with the SM prediction. For comparison, Fig. 国(b) shows the expected signal and background for a $\mathrm{BR}\left(t \rightarrow H^{+} b\right)=0.5$ and $m_{H^{ \pm}}=120 \mathrm{GeV}$, which clearly disagrees with the data.

Since the data agree with SM predictions, 95\% CL limits on $\mathrm{BR}\left(t \rightarrow H^{ \pm} b\right)$ are calculated assuming that $\mathrm{BR}\left(H^{+} \rightarrow \tau \nu_{\tau}\right)=1$. The limits shown in Fig. 目(c), are calculated using the modified frequentist approach [14] for $\mathcal{D}>0.55$ for the 3 jets events, and $\mathcal{D}>0.6$ for the $>3$ jets events. The region of small $\mathcal{D}$ is used to normalize the $W+$ jets background.
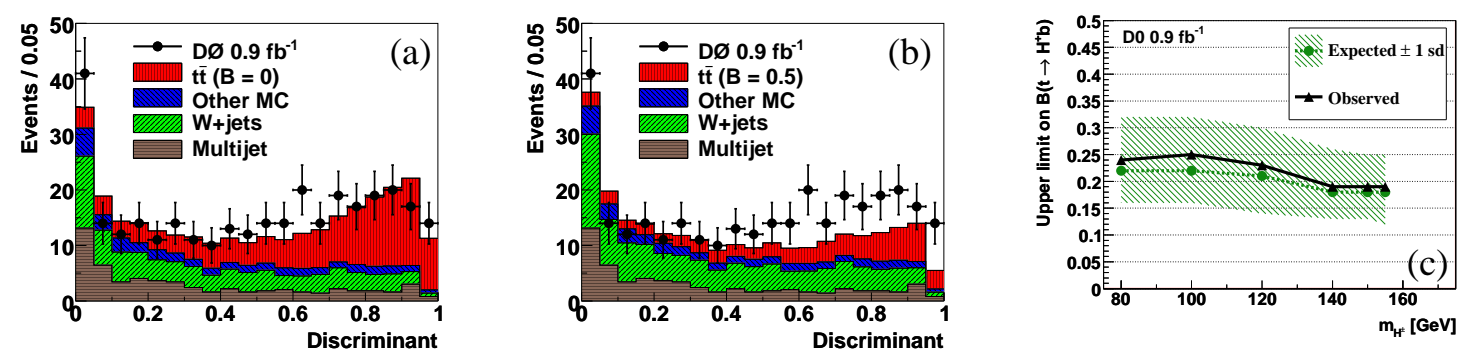

Figure 2: The discriminant for $M_{H^{ \pm}}=120 \mathrm{GeV}$, assuming (a) $\mathrm{BR}\left(t \rightarrow H^{+} b\right)=0$, and (b) $\mathrm{BR}\left(t \rightarrow H^{+} b\right)=$ 0.5. (c) The expected (assuming no contribution beyond SM) and observed $95 \%$ CL limits for $\mathrm{BR}\left(t \rightarrow H^{+} b\right)$.

\subsection{D0: Ratios of Production Cross Sections}

A powerful technique of searching for evidence of non-SM decays of $t$ quark is to calculate the ratios of $\sigma_{t \bar{t}}$ measured in different final states. What is measured is $\mathrm{BR} \cdot \sigma_{t \bar{t}}$, which can be divided by the appropriate SM value of BR to derive $\sigma_{t \bar{t}}$. If only SM decays are realized, then this defines the true $\sigma_{t \bar{t}}$. If there are non-SM contributions, the extracted values of $\sigma_{t \bar{t}}$ will depend on the final state. The ratios of $\sigma_{t \bar{t}}$ measured in different final states can therefore provide evidence for, or used to calculate limits on possible non-SM components in $t$ quark decays.

The D0 collaboration has extracted two ratios of $\sigma_{t \bar{t}}\left(R_{\sigma}\right)$ measured in different final states using $1 \mathrm{fb}^{-1}$ of integrated luminosity [15]. The first of these is measured in the $\ell^{ \pm} \ell^{\mp}$ relative to the $\ell^{ \pm}+$jets final states. This ratio is used to search for leptophobic decays, $\operatorname{BR}\left(H^{+} \rightarrow c \bar{s}\right) \approx 1$, that can occur in multi-Higgs-doublet models [16], and in the MSSM, if large radiative corrections arise from SUSY-breaking effects [17]. If $\mathrm{BR}\left(t \rightarrow H^{+} b\right)>0$, then both the number of $t \bar{t} \rightarrow \ell^{+} \ell^{-}$ and $t \bar{t} \rightarrow \ell^{ \pm}+$jets events will decrease relative to SM expectations, but the rate of decrease in $t \bar{t} \rightarrow \ell^{+}+$jets will be smaller than for $t \bar{t} \rightarrow \ell^{+} \ell^{-}$events leading to a value of $R_{\sigma}^{\ell \ell / \ell j}<1$.

The other measurement is of the ratio of $\sigma_{t \bar{t}}$ measured in the $\tau_{h}^{ \pm} \ell^{\mp}$ final states to the $\sigma_{t \bar{t}}$ measurement in the $\ell^{ \pm} \ell^{\mp}$ and $\ell^{ \pm}+$jets final states. This ratio is used to search for tauonic decays of $H^{ \pm}$. Under this assumption, the number of events measured in the $\tau_{h}^{ \pm} \ell^{\mp}$ final state will be greater than SM expectation, while the number of events for both $\ell^{ \pm} \ell^{\mp}$ and $\ell^{ \pm}+$jets final states will be 
smaller than SM expectations. The ratios $R_{\sigma}^{\tau_{h} \ell / \ell \ell}$ and $R_{\sigma}^{\tau_{h} \ell / \ell \text { jets }}$, whose mean we refer to as $R_{\sigma}^{\tau \ell / \ell \ell-\ell j}$, will therefore be $>1$. Correlations in systematic uncertainties among the individual $\sigma_{t \bar{t}}$, are estimated from pseudo-experiments. These sets are generated in MC, independently for the numerator and the denominator, by varying the number of signal and background events about their measured values, and the systematic uncertainties according to a Gaussian distribution. The simulation includes the $\ell^{ \pm} \ell^{\mp}$ events that mimic $\ell^{ \pm}+$jets, and the $\ell^{ \pm} \ell^{\mp}$ and $\ell^{ \pm}+$jets events that mimic $\tau_{h}^{ \pm} \ell^{\mp}$ events. The ratios are found to be $R_{\sigma}^{\ell \ell / \ell j}=0.86_{-0.17}^{+0.19}$ and $R_{\sigma}^{\tau \ell / \ell \ell-\ell j}=0.97_{-0.29}^{+0.32}$, which are consistent with the SM value of unity. Given the agreement with the SM for the different channels shown in Fig. 3 (a), including $\ell^{ \pm}+$jets with one and two $b$-jet tags, D0 calculates 95\% CL limits on non-SM contributions, as shown in Figs. 3 (b) and (c), using the frequentist approach of Ref [18].
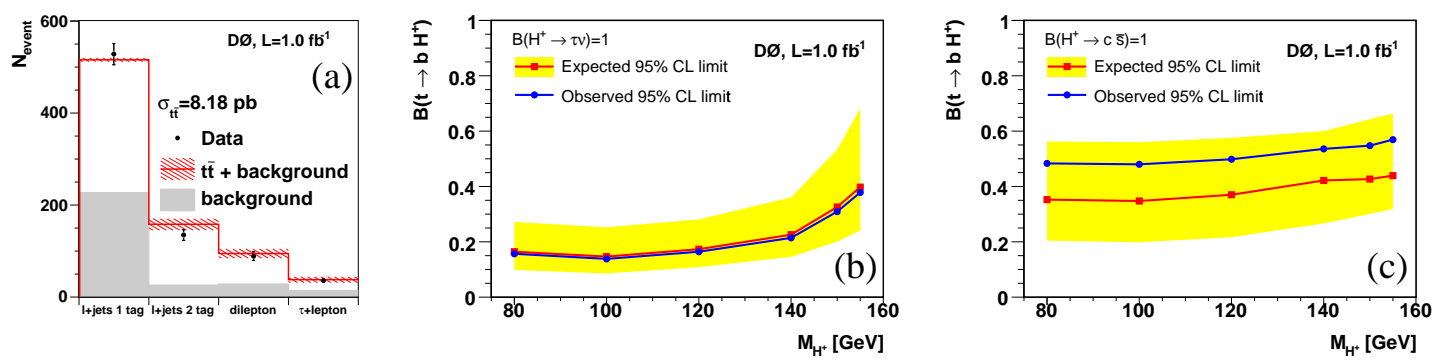

Figure 3: (a) Observed and SM number of events for different final states used to measure $\sigma_{t \bar{t}}$. The expected and observed $95 \% \mathrm{CL}$ limits for $\operatorname{BR}\left(t \rightarrow H^{+} b\right)$ in: (b) the tauonic and, (c) leptophobic models.

\subsection{D0: Global Fit}

An alternative to using the ratios of the measured $\sigma_{t \bar{t}}$, is to combine the measured $\sigma_{t \bar{t}}$ in a single global fit, and use the correlations among the different final states to search for evidence of non-SM decays of the $t$ quark. D0 has carried out this kind of search using $1 \mathrm{fb}^{-1}$ of integrated luminosity in the context of three models: 1) leptophobic, 2) tauonic, and 3) a combination of the two modes $\mathrm{BR}\left(H^{+} \rightarrow c \bar{s}\right)+\mathrm{BR}\left(H^{+} \rightarrow \tau^{+} v_{\tau}\right)=1[19]$.

The data samples are from the $\sigma_{t \bar{t}}$ measurements using the final states: $\ell^{ \pm}+$jets, $\ell^{ \pm} \ell^{\mp}$, and $\tau_{h}^{ \pm} \ell^{\mp}$, which are described in Refs. [15, 20]. The number of expected SM and $H^{ \pm}$events, is determined through a MC simulation of the experiment. The SM $t \bar{t}$ events are generated using the ALPGEN [21] event generator, and partons evolved using PYTHIA. The $H^{ \pm}$contribution to the $t \bar{t}$ decay is generated using PYTHIA. Both the SM and non-SM samples are processed using a simulation of the D0 detector and reconstructed with the same code as used for data. Figure 4 (a) shows the number of events in data, along with SM background and $t \bar{t}$ production. Since, the data and expectation agree with the SM, 95\% CL limits are calculated based on Ref. [18]. To account for statistical and systematic uncertainties and their correlations, D0 generates MC-experiments, the results from which are used to set bands of confidence for the observed branching ratio. The bands of confidence are then converted into $95 \%$ limits on $\operatorname{BR}\left(t \rightarrow H^{+} b\right)$, as a function of $M_{H^{ \pm}}$, for the observed values of branching ratio. Figures 4 show both the expected upper limits, with bands of \pm 1 standard deviation (sd), and the observed upper limits for the leptophobic and tauonic 
models, in (b) and (c), respectively. These limits are used to exclude regions in the $\left[M_{H^{ \pm}}, \tan \beta\right]$ plane for a number of MSSM scenarios [19].
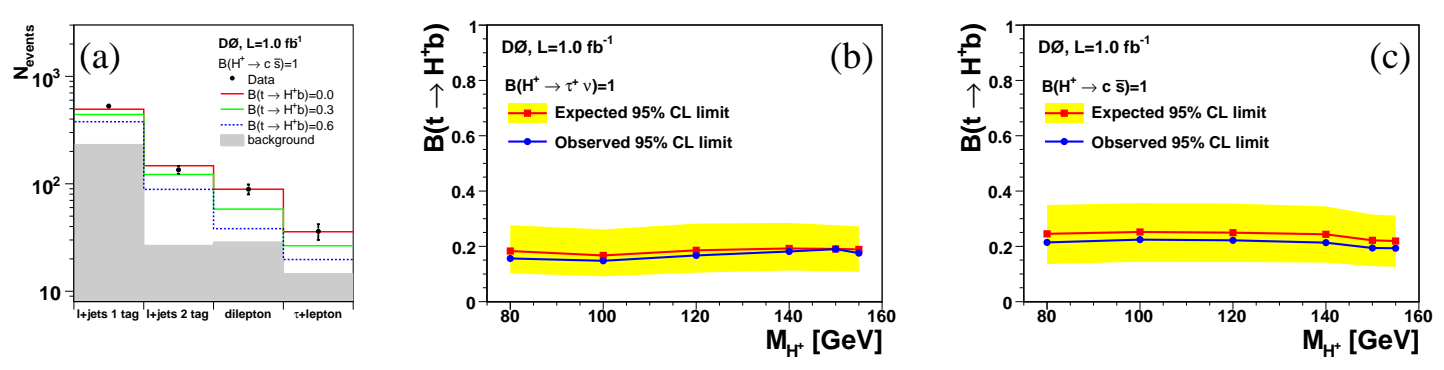

Figure 4: (a) Observed and expected number of events for different values of $\mathrm{BR}\left(t \rightarrow H^{+} b\right)$ in final states used to measure $\sigma_{t \bar{t}}$. The expected and observed $95 \% \mathrm{CL}$ upper limits for $\mathrm{BR}\left(t \rightarrow H^{+} b\right)$ in: (b) the tauonic model and (c) the leptophobic model for non-SM contributions to $t \bar{t}$ decay.

\section{Direct Searches}

Several direct searches for $H^{ \pm}$have been carried out at the Tevatron, and involve the modes $H^{+} \rightarrow c \bar{s}$ and $H^{+} \rightarrow t \bar{b}$. Another search involves extra tracks in SM $t$ quark decays that could signal a light $\mathrm{CP}$-odd Higgs boson $(A)$ that could have been missed in other analyses.

\subsection{CDF: $H^{+} \rightarrow c \bar{s}$}

Using $2.2 \mathrm{fb}^{-1}$ of integrated luminosity, CDF searches for $H^{ \pm}$in the context of a leptophobic model [22], using $t \bar{t} \rightarrow \ell^{ \pm}+$jets data selected by requiring lepton $p_{T}>20 \mathrm{GeV}$ with pseudorapidity $|\eta|<1$, and requiring the event to have $\not_{T}>20 \mathrm{GeV}$. The four jets with the most energy are required to have $E_{T}>20 \mathrm{GeV}$ and $|\eta|<2$, with at least two of the leading jets tagged as $b$ jets. Assuming $\sigma_{t \bar{t}}=6.7 \mathrm{pb}$ and $m_{t}=175 \mathrm{GeV}$, the $t \bar{t}$ signal to non- $t \bar{t}$ background is expected to be $11: 1$.

In the SM, the $\ell^{ \pm}+$jets final state is composed of two $b$ quark jets, and two light-quark jets ( $j j$ ) from the decay of the $W$. If the $t \rightarrow H^{+} b$ decay occurs, then, in addition to the $W$, another peak in the invariant mass will contribute to the light dijet mass spectrum. To improve the invariant mass resolution, a modified version of the kinematic fitter described in Ref. [23] is used to reconstruct the $t \bar{t}$ event. The $\ell^{ \pm}, \not_{T}, b$ quark jets and the light-quark jets are included in a global $\chi^{2}$, where the $\ell^{ \pm} B_{T}$ invariant mass is constrained to the $M_{W}$, and the $p_{T}$ of the final-state objects are allowed to vary within their measured uncertainties, as is the $p_{T}$ of the unclustered energy to improve the resolution in $Z_{T}$. Finally, the invariant masses of the two reconstructed $t$ quarks $\left(\ell^{ \pm} v_{\ell} b\right.$ and $\left.j j b\right)$ are required to have the same invariant mass but the $j j$ invariant mass is not constrained. The final dijet invariant-mass distribution is consistent with all dijets arising from $W$ decays, as can be seen in Fig. 自(a).

To extract the number of $W$ and $H^{ \pm}$events in data, a likelihood fit is performed to the data. Templates for the dijet mass distribution from $t \bar{t}$ decays generated using PYTHIA, and non-t $t \bar{t}$ background estimated from ALPGEN with parton evolution based on PYTHIA, are processed through a 
simulation of the CDF detector. The likelihood fit determines the normalization to the $W$ and $H^{ \pm}$ templates allowing the non- $t \bar{t}$ background to vary within the Gaussian uncertainties. The Bayesian 95\% CL upper limits on $\mathrm{BR}\left(t \rightarrow H^{+} b\right)$ assume a flat prior for $0 \leq \mathrm{BR}\left(t \rightarrow H^{+} b\right) \leq 1$ and treats all systematic uncertainties as nuisance parameters with Gaussian priors having means set to their nominal uncertainties. These are integrated over in the analysis. Figure 5 (b) show the $95 \% \mathrm{CL}$ upper limit on $\operatorname{BR}\left(t \rightarrow H^{+} b\right)$ for values $M_{H^{ \pm}}>M_{W}$. For $m_{H^{ \pm}} \approx M_{W}$, there is little sensitivity, so the points are excluded from the plot. For $M_{H^{ \pm}}<M_{W}$, the search assumes a generic scalar particle that can decay to $u \bar{d}$, which yields smaller upper limits, since there is only a small probability of tagging the light-quark jet as a $b$ quark.
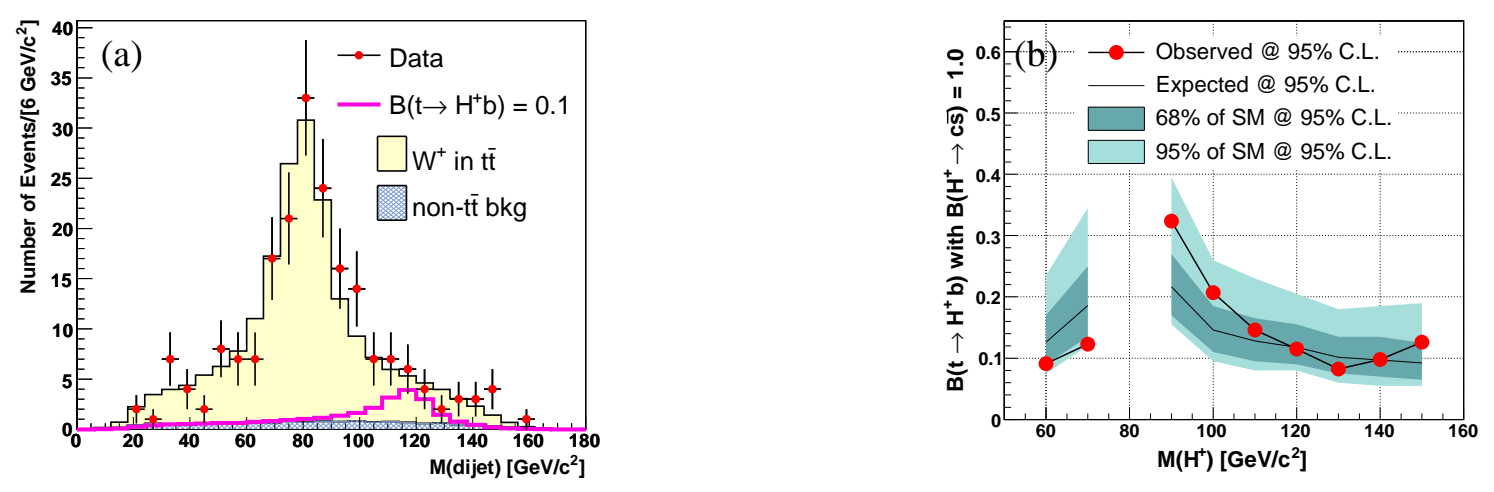

Figure 5: (a) The observed $j j$ mass distribution shown with the expected backgrounds from $t \bar{t}$ and non- $t \bar{t}$ sources. Also shown is the expected signal for $M_{H^{ \pm}}=120 \mathrm{GeV}$ and $\mathrm{BR}\left(t \rightarrow H^{+} b\right)=0.1$. (b) The $95 \% \mathrm{CL}$ upper limit on $\operatorname{BR}\left(t \rightarrow H^{+} b\right)$ as a function of $M_{H^{ \pm}}$.

\subsection{CDF: NMSSM}

The decay-mode-independent 95\% CL limit from LEP on a SM-like Higgs boson is $m_{h}>$ $82 \mathrm{GeV}$, which is in conflict with the MSSM predication of $m_{h}<82 \mathrm{GeV}$ [24. However, the nextto-minimal supersymmetric standard model (NMSSM), which adds a chiral-superfield singlet to the MSSM Higgs sector, increases $m_{h}$ beyond the current LEP limit. The NMSSM contains an additional CP-even and CP-odd Higgs boson, and an additional neutralino relative to the MSSM [25].

For $\tan \beta \lesssim 2.5$, the NMSSM $H^{ \pm}$comes within reach of observation at the Tevatron. CDF has carried out a direct search for this $H^{ \pm}$, assuming $M_{W} \lesssim M_{H^{ \pm}}<m_{t}-m_{b}$ and $m_{A}<2 m_{b}$ [26]. The $t$ quark can thereby be observed in final states containing $t \rightarrow H^{+} b \rightarrow W^{+(*)} A b$ decays in the mass range $4 \mathrm{GeV}<m_{A}<2 m_{b}$, with $\operatorname{BR}\left(A \rightarrow \tau^{+} \tau^{-}\right)>80 \%$. This decay mode could have been easily missed in previous searches, as the small mass of the $A$ leads to $\tau$ leptons with small $p_{T}$, and the final state taken as $W^{+} b$.

To focus on the final state containing the $A$, the CDF collaboration returned to the $\ell^{ \pm}+$jets sample of Ref. [27]. Assuming a $\tau_{h}$ decay of the $\tau$ lepton, each event is required to have one isolated charged track with $p_{T}>3 \mathrm{GeV}$, separated from the $\ell^{ \pm}$and jets by $\Delta R \equiv \sqrt{(\Delta \phi)^{2}+(\Delta \eta)^{2}}>0.4$, where $\phi$ is the azimuthal angle about the beam axis. The isolation requirement applied to the candidate track is that it have $>90 \%$ of the momentum of all tracks contained in a cone of $\Delta R>0.4$ about the candidate track. The background from the underlying event is calculated using three 
samples, 1) a dilepton sample of $Z$ candidates with one or two jets, 2) a $\ell^{ \pm}+$jets sample with one or two jets, and 3) a multijet sample. The $p_{T}$ spectrum of the isolated track for these different categories is found to be similar. Figure 6 (a) shows the expected background, the expected signal for a calculation with $M_{H^{ \pm}}=90 \mathrm{GeV}$ and $M_{A}=9 \mathrm{GeV}$, and the data. The data agree with the background, and 95\% CL limits are therefore calculated on $\operatorname{BR}\left(t \rightarrow H^{+} b\right)$ using the modified frequentist method, as shown in Fig. 6(b).
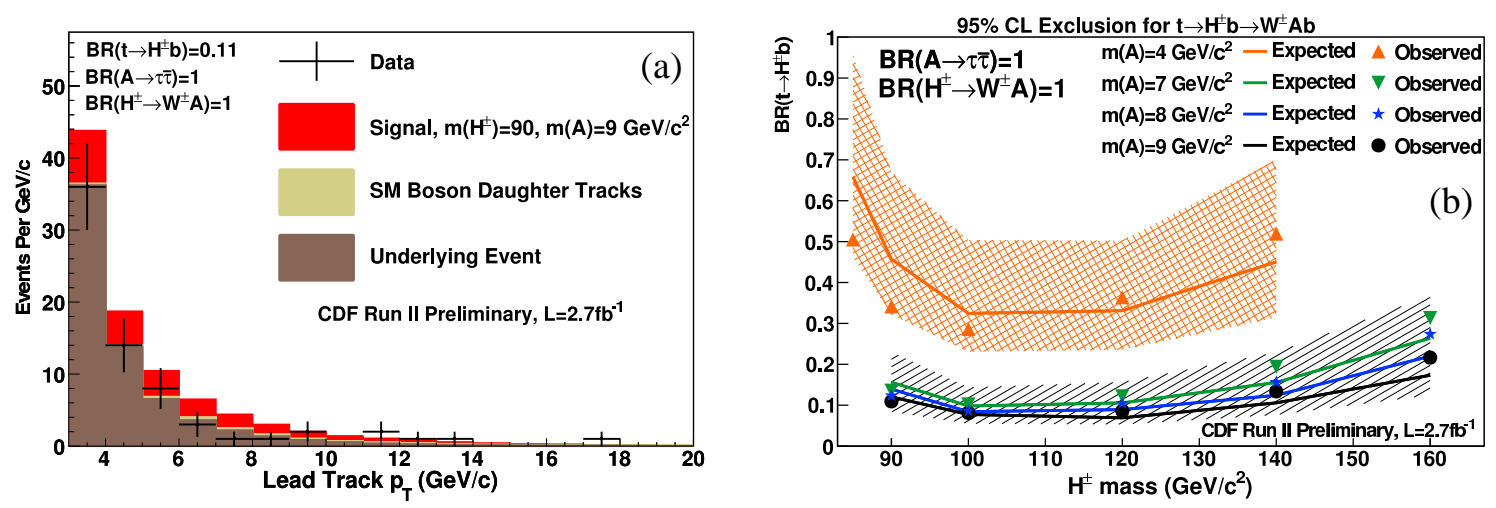

Figure 6: (a) The $p_{T}$ spectrum of the observed isolated track. (b) The expected and observed $95 \%$ CL upper limits on $\mathrm{BR}\left(t \rightarrow H^{+} b\right)$.

\subsection{D0: $H^{+} \rightarrow t \bar{b}$}

Using $1 \mathrm{fb}^{-1}$ of integrated luminosity, D0 has performed a search for direct $q \bar{q}^{\prime} \rightarrow H^{ \pm}$production for $M_{H^{ \pm}}>m_{t}$ [28]. In most such models, when $\tan \beta>1$, the $H^{ \pm}$decays predominately to $t \bar{b}$. The search is performed therefore in the final state of $H^{ \pm} \rightarrow t \bar{b} \rightarrow \ell^{ \pm}+Z_{T}+b \bar{b}$, which is the same as the final state in the $1 \mathrm{fb}^{-1}$ "single top" s-channel analysis [29]. The mass range for this search is restricted to $180<M_{H^{ \pm}}<300 \mathrm{GeV}$, which is larger than the smallest mass allowed for $H^{+} \rightarrow t \bar{b}$ and below the mass at which the cross section in most models is an order of magnitude smaller than its value at $180 \mathrm{GeV}$. This search, which complements other searches that have concentrated on $M_{H^{ \pm}}<m_{t}$, is performed using the same data sample and selection criteria as the "single top" analysis, except that the event must have exactly two jets, one or both of which are tagged as $b$ candidates [30]. After all selections are applied, the efficiency for signal varies from about $0.5 \%$ to $1.25 \%$ with increasing $M_{H^{ \pm}}$.

The $t \bar{b}$ invariant mass is used as the discriminating variable in the analysis. In terms of the observables, this corresponds to the invariant mass of jet1 (jet with the higher $p_{T}$ ), jet2 (the other jet) and the $W$ boson. The 4-vector of the $W$ boson is constructed from the $\ell^{ \pm} 4$-vector and the $Z_{T}$, constraining its mass to the known value of $M_{W}$. The longitudinal momentum $\left(p_{z}\right)$ of the neutrino is defined as the smallest absolute value of the two possible solutions for $p_{z}$. Figure 7 (a) shows the $t \bar{b}$ invariant mass, along with expectations for a Type-3 THDM coupling [㣙].

Given that the data are consistent with the SM expectations, 95\% CL Bayesian upper limits on charged Higgs production are calculated for the Type-1, 2 and 3 THDM discussed in Sect. 1. The effective Lagrangian for $H^{ \pm}$quark couplings in the THDM is proportional to the sum of leftand right-handed chiral couplings. Hence, to model $H^{ \pm}$production and calculate the efficiencies 

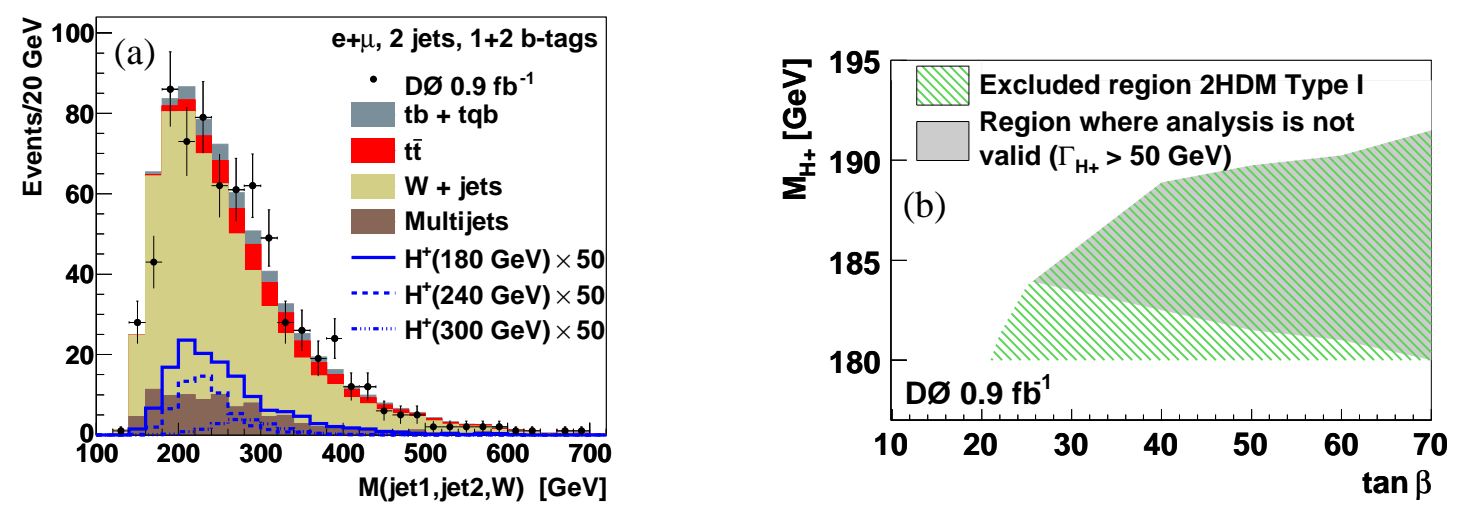

Figure 7: (a) Distributions in the invariant mass $M($ jet1, jet2, $W$ ) for expected signal and background compared to data. The signal corresponds to a Type-3 model. (b) The 95\% CL exclusion in the $\left[M_{H^{ \pm}}, \tan \beta\right]$ plane for a Type-1 THDM. The region with $\Gamma\left(H^{+} \rightarrow t \bar{b}\right)>50 \mathrm{GeV}$ corresponds to where the width of the charged Higgs width is significantly larger than detector resolution, and the results of the analysis not valid.

for detecting $H^{ \pm}$decays, events are generated using the COMPHEP program [31] for pure chiral states. These pure samples are then combined according to the strength of left- and right-handed couplings to simulate any desired version of the THDM.

The Bayesian limits on $\mathrm{BR}\left(H^{+} \rightarrow t \bar{b}\right) \cdot \sigma\left(q \bar{q}^{\prime} \rightarrow H^{+}\right)$are set by constructing a binned likelihood function, and assumes a flat prior for the signal cross section. All systematics and their correlations are taken into account in the calculated limits, which are in the range of 2 to $10 \mathrm{pb}$, and are larger than the expectation from the three THDM models, except for the Type- 1 model with values of $M_{H^{ \pm}}$close to $180 \mathrm{GeV}$. This is used to exclude regions in the $\left[M_{H^{ \pm}}, \tan \beta\right]$ plane, given in Fig. 团 (b). Results in the $\left[M_{H^{ \pm}}, \tan \beta\right]$ plane that correspond to $\Gamma\left(H^{+} \rightarrow t \bar{b}\right)$ significantly larger than detector resolution are not valid in this analysis.

\section{Summary}

Six recent results on $H^{ \pm}$searches from the CDF and D0 collaborations have been presented. These cover the $H^{ \pm}$mass range from $80 \mathrm{GeV}$ to $300 \mathrm{GeV}$. Both indirect searches, where deviations from SM expectations are used to seek evidence of $t \rightarrow H^{+} b$, and direct searches, where an enhancement in the mass spectrum or other direct evidence is used to establish a $H^{ \pm}$signal, provide null results.

\section{References}

[1] P.W. Higgs, Phys. Lett. 12, 132 (1964); Phys. Rev. Lett. 13, 508 (1964); Phys. Rev. 145, 1156 (1966); F. Englert and R. Brout, Phys. Rev. Lett. 13321 (1964); Phys. Rev. Lett. 13321 (1964);

G.S. Guralnik, C.R. Hagen and T.W.B. Kibble, Phys. Rev. Lett. 13585 (1964).

[2] CDF and D0 Collaborations, FERMILAB-CONF-10-257-E.

[3] R. Blair et al. (CDF Collaboration), FERMILAB-Pub-96/390-E (1996).

[4] V.M. Abazov et al. (D0 Collaboration), Nucl. Instrum. Methods A 565, 463 (2006). 
[5] V. Barger and R. J. N. Phillips, Phys. Rev. D 41, 884 (1990); J.F. Gunion, H.E. Haber, G. Kane, and S. Dawson, The Higgs Hunter's Guide, Frontiers in Physics (Addison-Wesley, Redwood City, Calif. 1990).

[6] S. Glashow and S. Weinberg, Phys. Rev. D 151958 (1977).

[7] H.-J. He and C.-P. Yuan, Phys. Rev. Lett. 83, 28 (1999).

[8] A. Abulencia et al. (CDF Collaboration) Phys. Rev. Lett. 96, 042003 (2006).

[9] J.S. Lee et al., Comput. Phys. Commun. 156, 283 (2004)

[10] T. Sjöstrand et al., Comput. Phys. Commun. 135, 238 (2001).

[11] R. Eusebi, Ph.D. thesis, University of Rochester, (2005).

[12] V. M. Abazov et al. (D0 Collaboration), Phys. Rev. D 80, 051107 (2009).

[13] V. M. Abazov et al. (D0 Collaboration), Phys. Rev. Lett. 100, 192004 (2008).

[14] T. Junk, Nucl. Instrum. Methods Phys. Res. A 434, 435 (1999).

[15] V.M. Abazov et al. (D0 Collaboration), Phys. Rev. D 80, 071102 (2009).

[16] Y. Grossman Nucl. Phys. B 425, 355 (1994); A.G. Akeroyd, hep-ph/9509203 (1995).

[17] M. Carena, S. Mrenna, and C.E.M. Wagner, Phys. Rev. D 62, 055008 (2000).

[18] G. J. Feldman and R. D. Cousins, Phys. Rev. D 57, 3873 (1998).

[19] V.M. Abazov et al. (D0 Collaboration), Phys. Lett. B 682278 (2009).

[20] V.M. Abazov et al. (D0 Collaboration), Phys. Lett. B 679, 177 (2009).

[21] M.L. Mangano et al., J. High Energy Phys. 07, 001 (2003).

[22] T. Aaltonen et al. (CDF Collaboration ) Phys. Rev. Lett. 103101803 (2009).

[23] A. Abulencia et al. (CDF Collaboration), Phys. Rev. D 73, 032003 (2006).

[24] G. Abbiendi et al. (OPAL Collaboration), Eur. Phys. J. C 27, 311 (2003).

[25] R. Dermisek and J. Gunion, Phys. Rev. D 79055014 (2009).

[26] (CDF Collaboration), CDF note 10104 (2010), http://www-cdf.fnal.gov/physics/new/top/2009/tprop/nMSSMhiggs/

[27] T. Aaltonen et al. (CDF Collaboration), Phys. Rev. Lett. 105, 012001 (2010).

[28] V.M. Abazov et al. (D0 Collaboration), Phys. Rev. Lett. 102, 191802 (2009).

[29] V.M. Abazov et al. (D0 Collaboration), Phys. Rev. Lett. 98, 181802 (2007); V.M. Abazov et al. (D0 Collaboration), Phys. Rev. D 78, 012005 (2008).

[30] V.M. Abazov et al. (D0 Collaboration), Nucl. Instrum. Methods Phys. Res. A 620, 490 (2010).

[31] E. Boos et al., (COMPHEP Collaboration), Nucl. Instrum. Methods Phys. Res. A 534, 250 (2004). 